\title{
A note on the weak sequential core of dynamic TU games
}

Citation for published version (APA):

Habis, H., \& Herings, P. J. J. (2010). A note on the weak sequential core of dynamic TU games.

METEOR, Maastricht University School of Business and Economics. METEOR Research Memorandum No. 022 https://doi.org/10.26481/umamet.2010022

Document status and date:

Published: 01/01/2010

DOI:

10.26481/umamet.2010022

Document Version:

Publisher's PDF, also known as Version of record

\section{Please check the document version of this publication:}

- A submitted manuscript is the version of the article upon submission and before peer-review. There can be important differences between the submitted version and the official published version of record.

People interested in the research are advised to contact the author for the final version of the publication, or visit the DOI to the publisher's website.

- The final author version and the galley proof are versions of the publication after peer review.

- The final published version features the final layout of the paper including the volume, issue and page numbers.

Link to publication

\footnotetext{
General rights rights.

- You may freely distribute the URL identifying the publication in the public portal. please follow below link for the End User Agreement:

www.umlib.nl/taverne-license

Take down policy

If you believe that this document breaches copyright please contact us at:

repository@maastrichtuniversity.nl

providing details and we will investigate your claim.
}

Copyright and moral rights for the publications made accessible in the public portal are retained by the authors and/or other copyright owners and it is a condition of accessing publications that users recognise and abide by the legal requirements associated with these

- Users may download and print one copy of any publication from the public portal for the purpose of private study or research.

- You may not further distribute the material or use it for any profit-making activity or commercial gain

If the publication is distributed under the terms of Article $25 \mathrm{fa}$ of the Dutch Copyright Act, indicated by the "Taverne" license above, 


\section{Maastricht University}

Helga Habis, P. Jean-J acques Herings

A Note on The Weak Sequential Core of Dynamic TU Games

RM/10/022

\section{METEOR}

Maastricht University School of Business and Economics

Maastricht Research School of Economics

of Technology and Organization

\section{P.O. Box 616}

NL - 6200 MD Maastricht

The Netherlands 


\title{
A Note on The Weak Sequential Core of Dynamic TU Games
}

\author{
Helga Habis* and P. Jean-Jacques Herings ${ }^{\dagger}$
}

January 15, 2010

\begin{abstract}
This paper addresses a problem with an argument in Kranich, Perea, and Peters (2005) supporting their definition of the Weak Sequential Core and their characterization result. We also provide the remedy, a modification of the definition, to rescue the characterization.
\end{abstract}

Keywords: cooperative games, dynamic games, core

JEL Classification: C71, C73

\section{Introduction}

Kranich, Perea, and Peters (2005) presents the concept of the Weak Sequential Core and its characterization for dynamic TU games without uncertainty. The main idea is that coalitions can only deviate from a given allocation if this deviation is credible; i.e. there is no sub-coalition at any date who can further improve upon it. Our focus here will be a reformulation of the idea of credibility.

By means of an example we argue that there is a problem in the argument of Kranich, Perea, and Peters (2005) supporting their definition of the Weak Sequential Core. We also provide a remedy, a modification of the definition, to rescue their results.

The outline of the paper is as follows. We specify the setup of the dynamic TU-game in Section 2. Next we provide the definition of the Weak Sequential Core following Kranich, Perea, and Peters (2005) in Section 3. We proceed with showing the problem with this definition in Section 4 and providing a remedy to it in Section 5.

${ }^{*}$ Department of Economics, Universiteit Maastricht, P.O. Box 616, 6200 MD, Maastricht, The Netherlands. E-mail: H.Habis@maastrichtuniversity.nl.

${ }^{\dagger}$ Department of Economics, Universiteit Maastricht, P.O. Box 616, 6200 MD, Maastricht, The Netherlands. E-mail: P.Herings@maastrichtuniversity.nl. The author would like to thank the Netherlands Organisation for Scientific Research (NWO) for financial support. 


\section{Preliminaries}

In this section we provide the basic definitions and the setting of the game played.

A TU-game is a pair $(N, w)$, where $N=\{1,2, \ldots, n\}$ is the set of players and $w: 2^{N} \rightarrow$ $\mathbb{R}$ is a characteristic function which assigns to each coalition $C \subset N$ its worth $w(C)$, with the convention that $w(\emptyset)=0$. The set of all TU-games with player set $N$ is denoted by $\mathcal{G}$.

We consider a sequence of TU-games, played in time-periods $t \in \mathcal{T}=\{1, \ldots, T\}$, by the players in $N$. Now the characteristic function $w_{t}: 2^{N} \rightarrow \mathbb{R}$ assigns to each coalition $C \subset N$ in each time-period $t$ its worth $w_{t}(C)$, with $w_{t}(\emptyset)=0$. Each player $i \in N$ has an intertemporal utility function, $u^{i}: \mathbb{R}^{T} \rightarrow \mathbb{R}$ which assigns to every payoff stream $x^{i}=\left(x_{1}^{i}, \ldots, x_{T}^{i}\right) \in \mathbb{R}^{T}$ of player $i$ a utility level $u^{i}\left(x^{i}\right)$. A dynamic TU-game is therefore defined as follows.

Definition 2.1. A dynamic TU-game, denoted by $\Gamma$, is a tuple $(N, \mathcal{T}, w, u)$, where $w=$ $\left(w_{1}, \ldots, w_{T}\right)$ and $u=\left(u^{1}, \ldots, u^{n}\right)$.

The utility function $u^{i}$ is assumed to be continuous, weakly increasing in each coordinate, time-separable, and satisfies

$$
\lim _{x_{t}^{i} \rightarrow-\infty} u^{i}\left(\bar{x}^{i} \backslash x_{t}^{i}\right)=-\infty
$$

where $\bar{x}^{i} \backslash x_{t}^{i}$ denotes the allocation where the period-t component of the allocation $\bar{x}^{i}$ is replaced by $x_{t}^{i}$.

The distribution of worth if the grand-coalition is formed is called an allocation; $x=$ $\left(x^{1}, \ldots, x^{n}\right) \in \mathbb{R}^{\mathcal{T} \times N}$. The period- $t$ component of the allocation is $x_{t}=\left(x_{t}^{1}, \ldots, x_{t}^{n}\right) \in \mathbb{R}^{N}$.

We introduce some useful notation to be able to define the subgame of a dynamic TUgame. Let $t^{+} \subset \mathcal{T}$ denote the set of time-periods from $t$ onwards, so $t^{+}=\{t, \ldots, T\}$. An allocation stream in time-periods from $t$ onwards is denoted $x_{t^{+}}$, and player $i$ 's payoff stream is $x_{t^{+}}^{i}$. Similarly, we use the notation for the characteristic function; $w_{t^{+}}=\left(w_{t}, \ldots, w_{T}\right)$.

We will use the following property of the utility functions. Since every utility function is assumed to be time-separable, there is a utility function $u_{t}^{i}: \mathbb{R}^{t^{+}} \rightarrow \mathbb{R}$ for each period $t$ such that for every $\left(x_{1}^{i}, \ldots, x_{t-1}^{i}\right)$ and every pair $y_{t^{+}}^{i}, \tilde{y}_{t^{+}}^{i}$ we have

$$
u^{i}\left(x_{1}^{i}, \ldots, x_{t-1}^{i}, y_{t^{+}}^{i}\right) \geq u^{i}\left(x_{1}^{i}, \ldots, x_{t-1}^{i}, \tilde{y}_{t^{+}}^{i}\right)
$$

if and only if

$$
u_{t}^{i}\left(y_{t^{+}}^{i}\right) \geq u_{t}^{i}\left(\tilde{y}_{t^{+}}^{i}\right)
$$

Let $u_{t}$ denote the utility functions $u_{t}=\left(u_{t}^{i}\right)_{i \in N}$, the collection of individual utility functions from $t$ onwards.

Now the subgame of a dynamic TU-game can be defined as follows.

Definition 2.2. The subgame of a dynamic TU-game $\Gamma$ starting at time period $t$ is the dynamic TU-game $\Gamma_{t}=\left(N, t^{+}, w_{t^{+}}, u_{t^{+}}\right)$. 
C.

For a dynamic TU-game $\Gamma,(\Gamma, C)$ will denote the restriction of the game to coalition

The central question in a TU-game is how to distribute the worth $w(C)$ of a coalition $C$ among its members if the coalition is formed. An allocation for a coalition $C$ in $\Gamma$ is a matrix $x^{C}=\left(x^{i}\right)_{i \in C} \in \mathbb{R}^{\mathcal{T} \times C}$. The allocation for a coalition $C$ at time-period $t$ is $x_{t}^{C} \in \mathbb{R}^{C}$ and an allocation stream for coalition $C$ in time-periods from $t$ onwards is denoted $x_{t^{+}}^{C}$. The total amount of payoff of coalition $C$ in time-period $t$ is $x_{t}(C)=\sum_{i \in C} x_{t}^{i}$, where $x_{t}^{i}$ is player $i$ 's share. The total payoff stream for the coalition from $t$ onwards is denoted by $x_{t^{+}}(C)$.

Definition 2.3. An allocation $\bar{x} \in \mathbb{R}^{\mathcal{T} \times N}$ is efficient in the game $\Gamma$ if

$$
\bar{x}(N)=w(N) .
$$

Note that this concept says more than the usual efficiency or feasibility conditions in TU games, since it requires $\sum_{i \in N} x_{t}^{i}=w_{t}(N)$ to hold for all time-periods $t \in \mathcal{T}$.

We study which allocations are stable in a game $\Gamma$. In general, a given allocation $\bar{x}$ is stable if there is no time-period $t \in \mathcal{T}$ and no coalition $C \subset N$ which has a profitable deviation from $\bar{x}$ at time-period $t$. There are various ways in which the notion of profitable deviation might be formulated. Here we concentrate on profitable deviations related to the Weak Sequential Core.

\section{The Weak Sequential Core}

In this section we reproduce the definition of the Weak Sequential Core following Kranich, Perea, and Peters (2005).

Definition 3.1. An allocation $x_{t^{+}}^{C} \in \mathbb{R}^{t^{+} \times C}$ is feasible for a coalition $C$ at time-period $t$ in the game $\Gamma$ if

$$
x_{t^{+}}(C)=w_{t^{+}}(C) .
$$

Note again that this concept says that the allocation must be feasible for coalition $C$ in every time-period; it requires $\sum_{i \in C} x_{t^{\prime}}^{i}=w_{t^{\prime}}(C)$ to hold for all time-periods $t^{\prime}$ from $t$ onwards.

Definition 3.2. Let some allocation $\bar{x}$ be given. A coalition $C$ can deviate from $\bar{x}$ at a given time period $t \in \mathcal{T}$ if there exists a feasible allocation $x_{t^{+}}^{C}$ for coalition $C$ at $t$ such that

$$
u_{t}^{i}\left(x_{t^{+}}^{i}\right)>u_{t}^{i}\left(\bar{x}_{t^{+}}^{i}\right) \text {, for all } i \in C .
$$

Since the utility functions are time-separable, the improvement in time-period $\bar{t}$ is independent of the payoffs received before $\bar{t}$. Note also that we implicitly assume that once a coalition deviates, it can no longer collaborate with players outside the coalition for the rest of the time. 
Definition 3.3. A deviation $x_{t^{+}}^{C}$ as in Definition 3.2 is credible if there is no sub-coalition $C^{\prime} \subset C$ and time-period $t^{\prime} \geq t$ such that $C^{\prime}$ has a counter-deviation at $t^{\prime}$ from $x_{t^{+}}^{C}$, i.e. a feasible allocation stream $\hat{x}_{t^{\prime+}}$ for $C^{\prime}$ with $u_{t^{\prime}}^{i}\left(\hat{x}_{t^{\prime+}}\right)>u_{t^{\prime}}^{i}\left(x_{t^{\prime+}}\right)$ for all $i \in C^{\prime}$.

Kranich, Perea, and Peters (2005) define the Weak Sequential Core as follows.

Definition 3.4. The Weak Sequential Core of the game $\Gamma$, denoted by $\operatorname{WSC}(\Gamma)$, is the set of feasible allocations $\bar{x}$ for the grand coalition from which no coalition ever has a credible deviation.

\section{Problem with the implications of $\mathrm{WSC}(\Gamma)$}

In this section we argue that if one follows the definitions of Section 3, the results of Kranich, Perea, and Peters (2005) may not hold. In particular we present a counter-example to their first result, Lemma 1.

First we re-state Lemma 1. Then we give an example, which proves Lemma 1 to be incorrect.

Lemma 1. Let $\Gamma$ be a dynamic TU-game and $\bar{x}$ a feasible allocation for $N$. Then the following two statements are equivalent.

(a) $\bar{x} \in \operatorname{WSC}(\Gamma)$,

(b) $\bar{x}$ is such that $\bar{x}_{2^{+}} \in \mathrm{WSC}\left(\Gamma_{2}\right)$ and there is no $C \subset N$ and allocation $x^{C}$ such that $x(C)=w(C), x_{2^{+}}^{C} \in \operatorname{WSC}\left(\Gamma_{2}, C\right)$ and $u^{i}\left(x^{i}\right)>u^{i}\left(\bar{x}^{i}\right)$ for all $i$ in $C$.

Example 4.1. Consider a game with two time-periods and two players; $\mathcal{T}=\{1,2\}$ and $N=\{1,2\}$. Let the stage games be given by

$$
\begin{aligned}
& w_{1}(\{1\})=0.9, \quad w_{1}(\{2\})=0, \quad w_{1}(\{1,2\})=1 \\
& w_{2}(\{1\})=0, \quad w_{2}(\{2\})=0.9, \quad w_{2}(\{1,2\})=1 .
\end{aligned}
$$

Let the utility functions be

$$
u^{i}\left(x^{i}\right)=1 / 2\left(1-e^{-x_{1}^{i}}\right)+1 / 2\left(1-e^{-x_{2}^{i}}\right)
$$

for both players.

Consider the following allocation

$$
\bar{x}=\left(\bar{x}^{1}, \bar{x}^{2}\right)=\left(\begin{array}{ll}
1 & 0 \\
0 & 1
\end{array}\right)
$$

This allocation would give both players a utility of $u^{i}\left(\bar{x}^{i}\right) \approx 0.3161$.

It is clear that no singleton coalition can deviate from the given allocation at any time, and also that the coalition $\{1,2\}$ cannot block $\bar{x}$ at $t=2$, since it is not possible to increase 
the utility of both players simultaneously. Thus it only remains to check if $\{1,2\}$ has a credible deviation $x^{\{1,2\}}$ at $t=1$. For such a deviation it must hold that

$$
\begin{aligned}
& u^{1}\left(x^{1}\right)>u^{1}(1,0), \\
& u^{2}\left(x^{2}\right)>u^{2}(0,1) .
\end{aligned}
$$

To prevent counter-deviations by players 1 and 2 at $t=2$ it should also hold that

$$
\begin{aligned}
& x_{2}^{1} \geq 0, \\
& x_{2}^{2} \geq 0.9 .
\end{aligned}
$$

It follows from Inequality (5) and feasibility that $x_{2}^{1} \leq 0.1$. Using Inequality (2) we find that

$$
1 / 2\left(1-e^{-x_{1}^{1}}\right)+1 / 2\left(1-e^{-0.1}\right) \geq u^{1}(1,0),
$$

so, $x_{1}^{1} \geq 0.7699$. By feasibility we obtain $x_{1}^{2} \leq 0.2301$.

The deviation $x^{1,2}$ therefore satisfies

$$
\begin{aligned}
& u^{1}\left(x^{1}\right) \leq u^{1}(1,0.1) \approx 0.3636, \\
& u^{2}\left(x^{2}\right) \leq u^{2}(0.2301,1) \approx 0.4188 .
\end{aligned}
$$

It follows that $x^{\{1,2\}}$ cannot be a credible deviation, since $x^{\prime}$ defined by

$$
x^{\prime}=\left(\begin{array}{ll}
0.455 & 0.545 \\
0.455 & 0.545
\end{array}\right)
$$

is a counter-deviation for coalition $\{1,2\}$ at $t=1 ; u^{1}\left(x^{\prime 1}\right) \approx 0.3656$ and $u^{2}\left(x^{\prime 2}\right) \approx 0.4202$.

Although $x^{\prime}$ itself is not credible, player 2 can counter-deviate from it in time-period 2 , $x^{\prime}$ makes any possible deviation by coalition $\{1,2\}$ at $t=1$ not credible.

Thus the given allocation $\bar{x}$ is in the Weak Sequential Core of the game.

Now we will show, by means of Example 4.1, that $(a)$ of Lemma 1 does not imply $(b)$.

We have seen that

$$
\bar{x}=\left(\bar{x}^{1}, \bar{x}^{2}\right)=\left(\begin{array}{ll}
1 & 0 \\
0 & 1
\end{array}\right)
$$

is an element of the Weak Sequential Core, so $\bar{x}$ satisfies the conditions in (a). It is also clear that the first claim of $(b)$ holds; i.e. $\bar{x}_{2^{+}}=\bar{x}_{2}=(0,1)$ is in the Weak Sequential Core of the subgame starting in time-period 2. The rest of the claim however is not true. Consider the allocation $x^{\{1,2\}}$ defined by

$$
x^{\{1,2\}}=\left(\begin{array}{cc}
0.9 & 0.1 \\
0.1 & 0.9
\end{array}\right) \text {. }
$$

It holds that $x^{\{1,2\}}(\{1,2\})=w(\{1,2\}), x_{2}^{\{1,2\}} \in \operatorname{WSC}\left(\Gamma_{2},\{1,2\}\right), u^{1}\left(x^{1}\right)>u^{1}\left(\bar{x}^{1}\right)$ and $u^{2}\left(x^{2}\right)>u^{2}\left(\bar{x}^{2}\right)$, so $\bar{x}$ does not satisfy the conditions in (b). Thus it follows that $(a)$ does not imply $(b)$. 


\section{Remedy}

Part $(b)$ of Lemma 1 plays a very important role in the concept of Weak Sequential Core. Predtetchinski, Herings, and Perea (2006) take (b) of Lemma 1 to define the Weak Sequential Core in their work on exchange economies and also Kranich, Perea, and Peters (2005) think of this condition as a crucial property of the $\mathrm{WSC}(\Gamma)$.

In this section we modify the definition of the Weak Sequential Core. Following Definition 4 in Kranich, Perea, and Peters (2005) the authors argue that the existence of a deviation by $C^{\prime}$ implies the existence of a credible counter-deviation by some coalition $C^{\prime \prime}$, and so it is not necessary to require a counter-deviation by $C^{\prime}$ to be credible; supporting both their definition of the Weak Sequential Core and the proof of Lemma 1.

Their argument proceeds as follows. Suppose that $C^{\prime}$ has a counter-deviation $x^{\prime}$ from $x^{C}$ that is not credible. One may assume that $C^{\prime}$ itself does not have a counter-deviation from this deviation. Then there is a coalition $C^{\prime \prime} \subsetneq C^{\prime}$ that has a deviation $x^{\prime \prime}$ from $x^{\prime}$ at a time-period $t^{\prime \prime} \geq t^{\prime}$. Next they claim that if $x^{\prime \prime}$ is a credible deviation from $x^{\prime}$ then it is also a credible deviation from $\bar{x}$. However this claim does not hold.

Consider the counter-deviation $x^{\prime}$ of $C^{\prime}=\{1,2\}$ at $t=1$ in Example 4.1. This deviation is not credible, $C^{\prime}$ itself does not have a deviation from it and $C^{\prime \prime} \subsetneq C^{\prime}=\{2\}$ does. Player 2 could deviate in time-period 2 with $x_{2}^{\prime \prime}=w_{2}(\{2\})=0.9>0.545$. However, $x^{\prime \prime}$ is not a deviation from $\bar{x}_{2}^{2}=1$, providing a counter-example to the claim above.

The solution to the problem is to require the counter-deviation by $C^{\prime}$ in Definition 3.3 to be credible at its turn. We propose to use a notion of credibility similar in spirit to the one defined by Ray (1989), applied to our dynamic setting.

Definition 5.1 (Credible deviation for a singleton). Let some allocation $\bar{x}$ be given. A feasible allocation $x_{t^{+}}^{\{i\}}$ is a deviation from $\bar{x}$ for player $i \in N$ in time-period $t \in \mathcal{T}$ if $u_{t}^{i}\left(x_{t^{+}}^{i}\right)>u_{t}^{i}\left(\bar{x}_{t^{+}}^{i}\right)$. Such a deviation is always credible for a singleton coalition.

A 2-player coalition $C$ has a credible deviation $x_{t^{+}}^{C}$ at time-period $t$ if there is no singleton sub-coalition $C^{\prime} \subsetneq C$ and time $t^{\prime} \geq t$ such that $C^{\prime}$ has a credible deviation at $t^{\prime}$ from $x_{t^{+}}^{C}$. Therefore, recursively, a credible deviation for a coalition $C$ is defined as follows.

Definition 5.2 (Credible deviation). Let some allocation $\bar{x}$ be given. A feasible allocation $x_{t^{+}}^{C}$ is a deviation from $\bar{x}$ for coalition $C$ in time-period $t \in \mathcal{T}$ if $u_{t}^{i}\left(x_{t^{+}}^{i}\right)>u_{t}^{i}\left(\bar{x}_{t^{+}}^{i}\right)$, for all $i \in C$. Such an $x_{t^{+}}^{C}$ is a credible deviation for coalition $C$ at time-period $t$ if there is no sub-coalition $C^{\prime} \subsetneq C$ and time $t^{\prime} \geq t$ such that $C^{\prime}$ has a credible deviation at $t^{\prime}$ from $x_{t^{+}}^{C}$.

Now the definition of the Weak Sequential Core can be modified as follows.

Definition 5.3. The Weak Sequential Core of the game $\Gamma$, denoted by $\operatorname{WSC}(\Gamma)$, is the set of feasible allocations $\bar{x}$ for the grand coalition from which no coalition ever has a credible deviation. 
If one applies this modified definition of credibility to Example 4.1, $\bar{x}$ defined by

$$
\bar{x}=\left(\bar{x}^{1}, \bar{x}^{2}\right)=\left(\begin{array}{ll}
1 & 0 \\
0 & 1
\end{array}\right)
$$

is no longer an element of the Weak Sequential Core. Consider $x^{\{1,2\}}$ defined by

$$
x^{\{1,2\}}=\left(x^{1}, x^{2}\right)=\left(\begin{array}{cc}
0.9 & 0.1 \\
0.1 & 0.9
\end{array}\right) .
$$

We claim that $x^{\{1,2\}}$ is a credible deviation from $\bar{x}$ by coalition $\{1,2\}$ at $t=1$. Indeed, $u^{1}\left(x^{1}\right)>u^{1}\left(\bar{x}^{1}\right), u^{2}\left(x^{2}\right)>u^{2}\left(\bar{x}^{2}\right)$, and there is no deviation possible from $x^{\{1,2\}}$ by any singleton coalition at any time-period.

We show next that if one uses Definition 5.3 instead of Definition 3.4 to define the Weak Sequential Core, Lemma 1 is rescued.

First we introduce two lemmas, which will be useful for our proof of Lemma 1 under the modified definition of the Weak Sequential Core.

Lemma 5.4. If, for some $t \in \mathcal{T}, \bar{x}_{t^{+}} \in \mathrm{WSC}\left(\Gamma_{t}\right)$, then it holds that $\bar{x}_{t^{\prime+}} \in \operatorname{WSC}\left(\Gamma_{t^{\prime}}\right)$ for all $t^{\prime} \geq t$.

Lemma 5.4 follows immediately from the definition of the Weak Sequential Core, so the proof is omitted.

Lemma 5.5. Let $\bar{x}$ be an efficient allocation and let $x^{C}$ be a credible deviation from $\bar{x}$ by coalition $C$ at time-period $t=1$. Let $D$ be the set of credible deviations $y^{C}$ from $\bar{x}$ by $C$ at $t=1$ with the property that $u^{i}\left(y^{i}\right) \geq u^{i}\left(x^{i}\right)$ for all $i \in C$. It holds that the set $D$ is compact.

Proof. First we show that $D$ is closed. Consider a sequence $\left(y_{n}^{C}\right)_{n \in \mathbb{N}}$ with $y_{n}^{C} \in D$ converging to $\bar{y}^{C}$. We need to show that $\bar{y}^{C} \in D$. Suppose not, i.e. $\bar{y}^{C} \notin D$. Then either

(i) $\bar{y}^{C}$ is not a credible deviation from $\bar{x}$ by $C$ at $t=1$, or

(ii) it does not hold that $u^{i}\left(\bar{y}^{i}\right) \geq u^{i}\left(x^{i}\right)$ for all $i \in C$.

Clearly $\bar{y}^{C}$ is a deviation from $\bar{x}$ by $C$ at $t=1$, so if $\bar{y}^{C}$ is not a credible deviation, then there is a credible counter-deviation $\hat{y}_{t^{+}}^{C^{\prime}}$ from $\bar{y}^{C}$ by a sub-coalition $C^{\prime} \subsetneq C$ at $t \geq 1$. Since $u_{t}^{i}\left(\bar{y}_{t^{+}}^{i}\right)<u_{t}^{i}\left(\hat{y}_{t^{+}}^{i}\right)$ for all $i \in C^{\prime}$, there must be an $\hat{n}$ such that if $n>\hat{n}$ then for all $i \in C^{\prime}$, $u_{t}^{i}\left(y_{t^{+}, n}^{i}\right)<u_{t}^{i}\left(\hat{y}_{t^{+}}^{i}\right)$. But then $y_{n}^{C}$ cannot be a credible deviation from $\bar{x}$ by $C$ at $t=1$ either, thus $(i)$ cannot hold.

The continuity of $u^{i}$ implies $u^{i}\left(\bar{y}^{i}\right) \geq u^{i}\left(x^{i}\right)$ for all $i \in C$, thus (ii) cannot hold. Hence, $D$ is closed.

Now we show that $D$ is bounded. We define the set $D_{t}$ by

$$
D_{t}=\left\{y_{t^{+}} \in \mathbb{R}^{t^{+} \times C} \mid y_{t^{+}} \text {is a credible deviation from } \bar{x} \text { by } C \text { at } t\right\} .
$$

Notice that $D \subset D_{1}$, since $u^{i}\left(y^{i}\right) \geq u^{i}\left(x^{i}\right)$ for all $i \in C$ is not required for $y^{C}$ to belong to $D_{1}$. We use backwards induction to prove that $D_{1}$ is bounded, thereby completing the proof of the lemma. 
1. For $i \in C$ and $y_{T}^{C} \in D_{T}$ it holds that

$$
y_{T}^{i} \geq w_{T}(\{i\})
$$

since $y_{T}^{i}=w_{T}(\{i\})$ if $C=\{i\}$, and $\{i\}$ should not have a deviation from $y_{T}$ if $\{i\} \subsetneq C$. Furthermore, since $y_{T}^{C}(C)=w_{T}(C)$, we have

$$
y_{T}^{i} \leq w_{T}(C)-\sum_{i^{\prime} \neq i} w_{T}\left(\left\{i^{\prime}\right\}\right),
$$

showing that $D_{T}$ is bounded.

2. Assume $D_{t+1}$ is bounded for some $t \in \mathcal{T}$. Let $\underline{b}^{C}$ and $\bar{b}^{C}$ be such that $\underline{b}^{C} \leq y_{(t+1)^{+}}^{C} \leq$ $\bar{b}^{C}$, for all $y_{(t+1)^{+}}^{C} \in D_{t+1}$. We show that $D_{t}$ is bounded.

Suppose $D_{t}$ is not bounded from below. Then there exists a sequence $\left(y_{t^{+}, n}^{C}\right)_{n \in \mathbb{N}}$ with $y_{t^{+}, n}^{C} \in D_{t}$ such that, for some $i \in C$ and for some $t^{\prime} \geq t$,

$$
y_{t^{\prime}, n}^{i} \leq-n \text { for all } n \in \mathbb{N} .
$$

Since $y_{t^{+}, n}^{C} \in D_{t}$, it follows that $y_{(t+1)^{+}, n}^{C} \in D_{t+1}$, and so $\underline{b}^{C} \leq y_{(t+1)^{+}, n}^{C} \leq \bar{b}^{C}$, and therefore $t^{\prime}=t$. Then, given that the utility function is assumed to be time-separable and weakly increasing in each coordinate, it holds that

$$
u_{t}^{i}\left(y_{t^{+}, n}^{i}\right) \leq u_{t}^{i}\left(y_{t, n}^{i}, \bar{b}^{i}\right) .
$$

By Equation (1), we know that

$$
\lim _{n \rightarrow \infty} u_{t}^{i}\left(y_{t, n}^{i}, \bar{b}^{i}\right)=-\infty
$$

and so by (7), we get

$$
\lim _{n \rightarrow \infty} u_{t}^{i}\left(y_{t^{+}, n}^{i}\right)=-\infty
$$

At the same time, credibility of $y_{t^{+}, n}^{i}$ implies $u_{t}^{i}\left(y_{t^{+}, n}^{i}\right) \geq u_{t}^{i}\left(w_{t^{+}}(\{i\})\right)$, and we obtain a contradiction, so we have shown that $D_{t}$ is bounded from below. It follows that $D_{t}$ is also bounded from above, since $y_{t^{+}}(C)=w_{t^{+}}(C)$.

Now we prove Lemma 1, using Definition 5.3 as the definition of the Weak Sequential Core.

Proof. (of Lemma 1)

First we show that $(a)$ implies $(b)$. The first part of $(b)$ holds as stated in Lemma 5.4. We prove the second part by contradiction. Suppose there is $C \subset N$ and $x^{C}$ such that 
$x(C)=w(C), x_{2^{+}}^{C} \in \operatorname{WSC}\left(\Gamma_{2}, C\right)$ and $u^{i}\left(x^{i}\right)>u^{i}\left(\bar{x}^{i}\right)$ for all $i \in C$. We show that if such a deviation $x^{C}$ exists, then there also exists a credible deviation from $\bar{x}$, thus contradicting $(a)$. If $x^{C}$ is a credible deviation, then we are done, so suppose $x^{C}$ is not a credible deviation. Since $x_{2^{+}}^{C} \in \operatorname{WSC}\left(\Gamma_{2}, C\right)$, for $x^{C}$ not being credible, there must be a credible deviation $\hat{x}^{C^{\prime}}$ from $x^{C}$ at time-period $t=1$ by a sub-coalition $C^{\prime} \subsetneq C$. But then $\hat{x}^{C^{\prime}}$ is also a credible deviation from $\bar{x}$ at $t=1$ by $C^{\prime}$ since $u^{i}\left(\hat{x}^{i}\right)>u^{i}\left(x^{i}\right)>u^{i}\left(\bar{x}^{i}\right)$ for all $i \in C^{\prime}$.

We show next that $(b)$ implies $(a)$. Suppose $(a)$ does not hold. Since $\bar{x}_{2^{+}} \in \operatorname{WSC}\left(\Gamma_{2}\right)$ by assumption, for $\bar{x} \notin \mathrm{WSC}(\Gamma)$ to hold, there must be a credible deviation $x^{C}$ from $\bar{x}$ by a coalition $C$ at $t=1$. We will show that then there also exists a credible deviation $\bar{y}^{C}$ from $\bar{x}$ by coalition $C$ at $t=1$ such that $\bar{y}_{2^{+}}^{C}$ belongs to $\operatorname{WSC}\left(\Gamma_{2}, C\right)$, thereby violating $(b)$.

Let $D$ be the set of credible deviations $y^{C}$ from $\bar{x}$ by $C$ at $t=1$ with the property that $u^{C}\left(y^{C}\right) \geq u^{C}\left(x^{C}\right)$. Let $\bar{y}^{C}$ be a credible deviation solving

$$
\max _{y^{C} \in D} \sum_{i \in C} u^{i}\left(y^{i}\right)
$$

Since the allocation $x^{C}$ belongs to $D$, so $D$ is non-empty, and we know from Lemma 5.5 that $D$ is compact, the set of maximizers in (8) is non-empty.

We show that $\bar{y}_{2^{+}}^{C}$ belongs to $\operatorname{WSC}\left(\Gamma_{2}, C\right)$. Suppose $\bar{y}_{2^{+}}^{C} \notin \operatorname{WSC}\left(\Gamma_{2}, C\right)$. Then there is a credible deviation $\hat{y}_{t^{+}}^{C^{\prime}}$ from $\bar{y}_{2^{+}}^{C}$ by $C^{\prime} \subset C$ at $t \geq 2$. Since $\bar{y}^{C}$ is a credible deviation, it is not possible that $C^{\prime} \subsetneq C$, so $C^{\prime}=C$.

We show that $\tilde{y}^{C}=\left(\bar{y}_{1}^{C}, \hat{y}_{2^{+}}^{C}\right)$ belongs to $D$, so

(i) $u^{i}\left(\tilde{y}^{i}\right) \geq u^{i}\left(x^{i}\right)$, for all $i \in C$,

(ii) $\tilde{y}^{C}$ is a credible deviation from $\bar{x}$ by coalition $C$ at $t=1$.

Part $(i)$ follows from the time-separability of the utility functions, since we have that $u^{i}\left(\tilde{y}^{i}\right)>u^{i}\left(\bar{y}^{i}\right) \geq u^{i}\left(x^{i}\right)$, for all $i \in C$.

Suppose $(i i)$ does not hold. Since $u^{i}\left(\tilde{y}^{i}\right) \geq u^{i}\left(x^{i}\right)>u^{i}\left(\bar{x}^{i}\right)$, for all $i \in C$, for $\tilde{y}^{C}$ not to be a credible deviation from $\bar{x}$, there should be a coalition $C^{\prime} \subsetneq C$ with a credible deviation $z_{t^{\prime+}}^{C^{\prime}}$ from $\tilde{y}^{C}$ at $t^{\prime}$. This leads to a contradiction when $t^{\prime} \geq t$ since $\hat{y}_{t^{+}}^{C}$ is credible, and to a contradiction when $t^{\prime}<t$ since $\bar{y}^{C}$ is a credible. We have shown that $\tilde{y}^{C} \in D$. It follows that $\sum_{i \in C} u^{i}\left(\tilde{y}^{i}\right)>\sum_{i \in C} u^{i}\left(\bar{y}^{i}\right)$, which contradicts that $\bar{y}^{C}$ is a maximizer. We have shown that $\bar{y}_{2^{+}}^{C} \in \operatorname{WSC}\left(\Gamma_{2}, C\right)$.

\section{Conclusion}

The original definition of the Weak Sequential Core for dynamic transferable utility games as proposed in Kranich, Perea, and Peters (2005) is problematic since it is incompatible with the main characterization of the Weak Sequential Core. In turn, this characterization was used to define the Weak Sequential Core for economies with incomplete asset markets 
in Predtetchinski, Herings, and Perea (2006). We propose a modification of the original definition under which the desired equivalence with the main characterization of the Weak Sequential Core is rescued.

\section{References}

Kranich, L., A. Perea, and H. Peters (2005): "Core Concepts For Dynamic TU Games," International Game Theory Review, 7, 43-61.

Predtetchinski, A., P. Herings, and A. Perea (2006): "The Weak Sequential Core for Two-Period Economies," International Journal of Game Theory, 34, 55-65.

RAY, D. (1989): "Credible Coalitions and the Core," International Journal of Game Theory, 18, 185-187. 